\title{
EXTENSIONS QUADRATIQUES 2-BIRATIONNELLES DE CORPS TOTALEMENT RÉELS
}

\author{
Jean-François Jaulent et Odile Sauzet
}

\begin{abstract}
We characterize 2-birational CM-extensions of totally real number fields in terms of tame ramification. This result completes in this case a previous work on pro- $\ell$-extensions over 2 -rational number fields.
\end{abstract}

\section{Position du problème}

La notion de corps $S$-rationnel a été introduite dans [JSa], en liaison avec les résultats de [W1] et [W2], pour généraliser la notion de corps de nombres $\ell$-rationnel rencontrée implicitement dans des contextes variés par plusieurs auteurs puis explicitement définie et étudiée par $[\mathbf{M N}]$ d'une part et $[\mathbf{G J}]$ d'autre part (cf. $[\mathbf{J N}]$ ). Rappelons ce dont il s'agit: si $\ell$ est un nombre premier et $S$ un sous-ensemble non vide de l'ensemble $P l_{K}^{\ell}$ des places de $K$ au-dessus de $\ell$, on dit que le corps de nombres $K$ est $S$-rationnel lorsque le groupe de Galois $G_{K}=$ $\operatorname{Gal}\left(K^{\prime} / K\right)$ de sa pro- $\ell$-extension (galoisienne) $\ell$-ramifiée maximale est le pro- $\ell$-produit libre

$$
\left.G_{K} \simeq \underset{\substack{\mathfrak{p} \mid \ell \infty \\ \mathfrak{p} \notin S}}{* \mathcal{G}_{\mathfrak{p}}}\right) * \mathcal{F}
$$

des groupes de Galois locaux $\mathcal{G}_{\mathfrak{p}}=\operatorname{Gal}\left(\bar{K}_{\mathfrak{p}} / K_{\mathfrak{p}}\right)$ respectivement attachés aux pro- $\ell$-extensions maximales $\bar{K}_{\mathfrak{p}}$ des completés $K_{\mathfrak{p}}$ de $K$ aux places réelles ou $\ell$-adiques qui ne sont pas dans $S$, et d'un pro- $\ell$-groupe libre $\mathcal{F}$; dans ce cas, le nombre $f$ de générateurs de $\mathcal{F}$ est donné par la formule

$$
f=d-r-c-l+s+1,
$$

où $d$ est la somme des degrés locaux $d=\sum_{\mathfrak{l} \in S}\left[K_{\mathfrak{l}}: \mathbb{Q}_{\ell}\right]$ et $r, c, l, s$ sont respectivement les nombres de places réelles, complexes, $\ell$-adiques ou dans $S$ de $K$ (cf. [JSa, th. 2.7]). Lorsque $S$ est un singleton $\{\mathfrak{l}\}$, on parle de corps l-rationnel et si $P l_{K}^{\ell}$ est lui même un singleton, on dit tout simplement que $K$ est $\ell$-rationnel. Dans ce dernier cas, si $K$ contient en 
outre les racines $\ell$-ièmes de l'unité, la condition (i) ci-dessus a lieu si et seulement si le $\ell$-groupe $C \ell_{K}^{\prime}$ des $\ell$-classes de diviseurs de $K$ (i.e. ici le quotient du $\ell$-groupe des classes d'idéaux prises au sens restreint par le sous-groupe engendré par la classe de l'idéal premier au dessus de $\ell$ ) est trivial, ce qui s'écrit

$$
C \ell_{K}^{\prime}=1
$$

de sorte que la notion de $\ell$-rationalité coïncide alors avec celle de $\ell$-régularité introduite par Kummer dans l'étude des corps cyclotomiques $\mathbb{Q}\left[\zeta_{l}\right]$.

La question de la propagation de la $S$-rationalité dans une $\ell$-extension $L / K$ de corps de nombres a été complètement résolue dans [JSa] pour les $\ell$ impairs. Pour $\ell=2$, en revanche, et $L / K$ quadratique, il peut arriver que le corps de base $K$ soit l-rationnel en une place 2-adique décomposée dans l'extension $L / K$ et que $L$ soit l-rationnel pour chacune des deux places au-dessus de $\mathfrak{l}$ (on dit alors qu'il est birationnel), situation que les arguments de $[\mathbf{J S a}]$ ne permettent pas de traiter complètement.

L'objet de ce travail est précisement d'éclaircir ce point plus délicat, dans le cas particulier où le corps de base $K$ est totalement réel, en prenant appui sur la notion de classes logarithmiques exposée dans $[\mathbf{J} 1]$.

\section{Index des notations}

Nous utiliserons dans tout ce qui suit les notations de la théorie $\ell$-adique du corps de classes (avec ici $\ell=2$ ) telle qu'exposée dans [J]2]. En particulier si $K$ est un corps de nombres et $\mathfrak{p}$ une place de $K$, nous notons:

- $K_{\mathfrak{p}}$ le complété de $K$ en la place $\mathfrak{p}$;

$-\mathcal{R}_{\mathfrak{p}}=\varliminf_{\mathfrak{p}} K_{\mathfrak{p}}^{\times} / K_{\mathfrak{p}}^{\times 2^{n}}$ le compactifié 2 -adique de $K_{\mathfrak{p}}^{\times}$;

- $\tilde{\mathcal{U}}_{\mathfrak{p}}$ le sous-groupe des unités logarithmiques de $\mathcal{R}_{\mathfrak{p}}$, i.e. le noyau dans $\mathcal{R}_{\mathfrak{p}}$ de la valeur absolue 2 -adique $|\cdot|_{\mathfrak{p}}$;

- $\mu_{\mathfrak{p}}$ le sous-groupe de torsion de $\mathcal{R}_{\mathfrak{p}}$, i.e. le 2-groupe des racines de l'unité dans $K_{\mathfrak{p}}$;

- $\mathcal{J}_{K}=\prod_{\mathfrak{p}}^{\text {res }} \mathcal{R}_{\mathfrak{p}}$ le 2-groupe des idèles de $K$;

- $\tilde{\mathcal{J}}_{K}$ le noyau dans $\mathcal{J}_{K}$ de la formule du produit pour les valeurs absolues 2-adiques;

- $\tilde{\mathcal{U}}_{K}=\prod_{\mathfrak{p}} \tilde{\mathcal{U}}_{\mathfrak{p}}$ le sous-groupe des unités logarithmiques semi-locales;

- $\mathcal{R}_{K}=\mathbb{Z}_{2} \otimes_{\mathbb{Z}} K^{\times}$le 2-groupe des idèles principaux (plongé dans $\left.\mathcal{J}_{K}\right)$;

- $\tilde{\mathcal{E}}_{K}=\mathcal{R}_{K} \cap \tilde{\mathcal{U}}_{K}$ le sous-groupe des unités logarithmiques globales; 
- $\mathcal{E}^{\prime}{ }_{K}=\mathbb{Z}_{2} \otimes_{\mathbb{Z}} E^{\prime}{ }_{K}$ le sous-groupe construit sur les 2-unités (au sens ordinaire);

- $\mathcal{D}_{K}=\mathcal{J}_{K} / \tilde{\mathcal{U}}_{K}$ le 2-groupe des diviseurs logarithmiques de $K$;

- $\tilde{\mathcal{D}}_{K}=\tilde{\mathcal{J}}_{K} / \tilde{\mathcal{U}}_{K}$ le sous-groupe des diviseurs logarithmiques de degré nul;

- $\tilde{\mathcal{P}}_{K}$ le sous-groupe des diviseurs logarithmiques principaux, i.e. l'image canonique de $\mathcal{R}_{K}$ dans $\tilde{\mathcal{D}}_{K}$;

- $\tilde{C} \ell_{K}^{\prime}=\tilde{\mathcal{D}}_{K} / \tilde{\mathcal{P}}_{K}=\tilde{\mathcal{J}}_{K} / \mathcal{R}_{K} \tilde{U}_{K}$ le 2-groupe des classes logarithmiques;

- $C \ell_{K}^{\prime}=\mathcal{J}_{K} / \mathcal{R}_{K} \prod_{\mathfrak{p} \nmid 2 \infty} \mu_{\mathfrak{p}} \prod_{\mathfrak{r} \mid 2} \mathcal{R}_{\mathfrak{l}}$ le 2-groupe des 2-classes (au sens restreint).

Enfin pour une extension $L / K$ de corps de nombres, nous écrivons:

- $\mathcal{N}_{L / K}$ le sous-groupe de $\mathcal{R}_{K}$ formé des éléments qui sont une norme locale dans $L / K$ (lorsque $L / K$ est cyclique, c'est tout simplement le groupe $N_{L / K}\left(\mathcal{R}_{L}\right)$ des normes globales).

Conformément aux conventions de $[\mathbf{J} 2]$ nous réservons aux places finies le concept de ramification; si $\mathfrak{p}$ est une place réelle, nous parlons, s'il y a lieu, de complexification. De plus, toutes les extensions considérées ici étant des 2-extensions, la ramification est ipso facto modérée aux places étrangères à 2 et sauvage aux places 2 -adiques.

\section{Enoncé du résultat principal}

Nous supposons désormais que $\ell$ vaut 2 et que $L$ est une extension quadratique totalement imaginaire d'un corps $K$ totalement réel. Notre propos est de relier la 2-rationalité de $K$ avec la 2-birationalité de $L$. Rappelons ce que nous entendons pas là:

Définition 1 (cf. [JN, th. 1.2]). Un corps totalement réel $K$ est dit 2-rationnel lorsque sa 2-extension (galoisienne) 2-ramifiée $\infty$-décomposée maximale $K^{\prime}$ coïncide avec sa $\mathbb{Z}_{2}$-extension cyclotomique $K^{c}$, ce qui a lieu si et seulement si les deux conditions suivantes sont réunies:

(1a) $K$ admet une unique place 2 -adique $\mathfrak{l}$;

(1b) le 2-groupe $\mathcal{C} \ell_{K}^{\prime}$ des 2-classes est trivial (en d'autres termes ici le 2-sous-groupe de Sylow du groupe des classes d'idéaux au sens restreint de $K$ est engendré par l'image de la classe de $\mathfrak{l}$ ). 
Définition 2 (cf. [JSa, th. 1.11]). Un corps totalement imaginaire $L$ est dit 2-birationnel lorsqu'il est l-rationnel en chacune des places 2-adiques (en ce sens qu'il n'admet pas de 2-extension 2-ramifiée l-décomposée non triviale), ce qui a lieu si et seulement si les trois conditions suivantes sont vérifiées:

(2a) $L$ admet exactement 2 places 2 -adiques $\mathfrak{l}$ et $\mathfrak{l}^{\prime}$;

(2b) le 2-groupe $C \ell_{L}^{\prime}$ des 2-classes de diviseurs de $L$ est trivial (en d'autres termes ici le 2-sous-groupe de Sylow du groupe des classes d'idéaux de $L$ (au sens ordinaire comme au sens restreint) est engendré par les images de $\mathfrak{l}$ et de $\mathfrak{l}^{\prime}$ );

(2c) les plongements canoniques de $L^{\times}$dans $L_{\mathfrak{l}}^{\times}$et $L_{\mathfrak{l}^{\prime}}^{\times}$induisent des isomorphismes $\mathcal{E}_{L}^{\prime} \simeq \mathcal{R}_{l^{\prime}} \simeq \mathcal{R}_{\mathfrak{l}}$ du tensorisé 2 -adique du groupe des 2-unités de $L$ sur les compactifiés des groupes multiplicatifs des complétés de $L$ aux places 2 -adiques.

Introduisons maintenant la notion de place primitive: étant donné un corps de nombres $K$, notons provisoirement $K^{z}$ le compositum des $\mathbb{Z}_{2}$-extensions de $K$ et $K^{c}$ la $\mathbb{Z}_{2}$-extension cylotomique de $K$; dans [GJ] (cf. déf. 1.1) une place modérée de $K$ (i.e. une place finie étrangère à 2 ) est dite primitive lorsque son image dans $\operatorname{Gal}\left(K^{z} / K\right)$ par l'application d'Artin n'est pas un carré; elle est dite dans [J1] (cf. déf. 4.4) logarithmiquement primitive lorsque c'est son image dans $\operatorname{Gal}\left(K^{c} / K\right)$ qui n'est pas un carré. Il se trouve que, lorsque $K$ est totalement réel et vérifie la conjecture de Leopoldt en 2, le compositum $K^{z}$ des $\mathbb{Z}_{2}$-extensions se réduit à la $\mathbb{Z}_{2}$-extension cyclotomique $K^{c}$, de sorte que les deux notions coïncident. Nous adopterons donc dans ce qui suit la convention suivante:

Définition 3. Soit $K$ un corps de nombres totalement réel qui satisfait la conjecture de Leopoldt en $\ell=2$. Nous dirons qu'une place modérée $\mathfrak{p}$ de $K$ (i.e. une place finie ne divisant pas 2) est (relativement au nombre premier 2)

- primitive, lorsque son image dans le groupe procyclique $\operatorname{Gal}\left(K^{c} / K\right)$ n'est pas un carré, autrement dit lorsqu'elle n'est pas décomposée dans la $\mathbb{Z}_{2}$-extension cyclotomique $K^{c} / K$;

- semi-primitive, lorsque son image est un carré mais non une puissance 4-ième, autrement dit lorsqu'elle se décompose dans le premier étage de la $\mathbb{Z}_{2}$-extension cyclotomique $K^{c} / K$ mais pas audelà.

Cela étant posé, le résultat principal de cet article est le suivant: 
Théorème 4. Soit $L / K$ une extension quadratique totalement imaginaire d'un corps de nombres totalement réel. Les assertions suivantes sont équivalentes:

(i) le corps L est 2-birationnel;

(ii) le corps $K$ est 2-rationnel, son unique place 2-adique est décomposée dans $L / K$ et l'extension $L / K$ est ramifiée modérément soit en une place semi-primitive $\mathfrak{p}$ soit en deux places primitives $\mathfrak{p}$ et $\mathfrak{q}$.

Exemple. Pour $K=\mathbb{Q}$ et $L=\mathbb{Q}[\sqrt{-d}]$, on retrouve ainsi la classification des corps quadratiques imaginaires 2-birationnels donnée dans [JSa] (cf. cor. 1.12): ce sont les corps $\mathbb{Q}[\sqrt{-p}]$ avec $p \equiv 7[\bmod 16]$ premier et les corps $\mathbb{Q}[\sqrt{-p q}]$ avec $p \equiv-q \equiv 3[\bmod 8]$ premiers.

Remarque. Le théorème de densité de Čebotarev montre alors que tout corps de nombres 2-rationnel totalement réel possède une infinité d'extensions quadratiques totalement imaginaires qui sont 2-birationnelles.

\section{Interprétation logarithmique de la 2-rationalité}

Commençons par rappeler la construction du 2-groupe des classes logarithmiques (cf. $[\mathbf{J} 1]$ pour plus de détails). Soit

$$
\tilde{\mathcal{J}}_{L}=\left\{\mathfrak{x}=\left.\left(x_{\mathfrak{P}}\right)_{\mathfrak{P}} \in \mathcal{J}_{L}\left|\prod_{\mathfrak{P}}\right| x_{\mathfrak{P}}\right|_{\mathfrak{P}}=1\right\}
$$

le noyau dans le 2-groupe des idèles $\mathcal{J}_{L}=\prod_{\mathfrak{P}}^{\text {res }} \mathcal{R}_{\mathfrak{P}}$ de la formule du produit pour les valeurs absolues 2 -adiques et

$$
\tilde{\mathcal{U}}_{L}=\left\{\mathfrak{x}=\left.\left(x_{\mathfrak{P}}\right)_{\mathfrak{P}} \in \mathcal{J}_{L}|| x_{\mathfrak{P}}\right|_{\mathfrak{P}}=1, \forall \mathfrak{P} \in P l_{L}\right\}
$$

le sous-groupe des unités logarithmiques locales; le quotient $\tilde{\mathcal{D}}_{L}=\tilde{\mathcal{J}}_{L} / \tilde{\mathcal{U}}_{L}$ est, par définition, le 2-groupe des diviseurs logarithmiques de $L$ et son quotient $\tilde{C} \ell_{L}^{\prime}=\tilde{\mathcal{D}}_{L} / \tilde{\mathcal{P}}_{L}=\tilde{\mathcal{J}}_{L} / \mathcal{R}_{L} \tilde{U}_{L}$ par l'image canonique $\tilde{\mathcal{P}}_{L} \mathrm{du}$ 2-groupe $\mathcal{R}_{L}=\mathbb{Z}_{2} \otimes_{\mathbb{Z}} L^{\times}$des idèles principaux est le 2 -groupe des classes logarithmiques du corps $L$.

Dans la correspondance du corps de classes, le groupe d'idèles $\tilde{\mathcal{J}}_{L}$ est le groupe de normes associé à la $\mathbb{Z}_{2}$-extension cyclotomique $L^{c}$ de $L$ et son sous-groupe $\tilde{\mathcal{U}}_{L} \mathcal{R}_{L}$ est, lui, le sous-groupe de normes associé à la pro-2-extension abélienne localement cyclotomique maximale $L^{\text {lc }}$ de $L$ (i.e. à la plus grande 2-extension abélienne de $L$ qui est complètement décomposée sur $L^{c}$ en chacune des ses places), de sorte que le quotient $\tilde{C} \ell_{L}$ s'identifie au groupe de $\operatorname{Galois} \operatorname{Gal}\left(L^{l c} / L^{c}\right)$. Lorsque $\tilde{C} \ell_{L}$ est trivial, $L^{l c}$ coïncide avec $L^{c}$ et on dit que $L$ est 2-logarithmiquement principal. 
Cela étant nous allons déduire le théorème 4 du critère suivant de birationalité:

Proposition 5. Soit $L / K$ une extension quadratique totalement imaginaire d'un corps de nombres totalement réel. Il a alors équivalence entre:

(i) le corps L est 2-birationnel;

(ii) le corps $K$ est 2 -rationnel, l'extension $L / K$ est 2 -décomposée mais ramifiée modérement en au moins une place finie et le corps $L$ est 2-logarithmiquement principal.

Commençons pour cela par établir un lemme:

Lemme 6. Si $L$ est un corps 2-birationnel extension quadratique d'un sous-corps $K$ totalement réel, celui-ci est 2-rationnel et l'extension $L / K$ est décomposée au-dessus de 2 et ramifiée modérément en au moins une place finie.

Preuve du lemme: Vérifions d'abord que les places 2-adiques sont décomposée dans $L / K$. Dans le cas contraire, puisque $L$ supposé 2-birationnel possède exactement deux places 2 -adiques, disons $\mathfrak{L}$ et $\mathfrak{L}^{\prime}$, le sous-corps $K$ posséderait aussi deux places 2 -adiques disons $\mathfrak{l}$ et $\mathfrak{l}^{\prime}$, et les résultats de $[\mathbf{J S a}]$ (cf. cor. 2.11) montrent que, puisque $L$ est rationnel en $\mathfrak{L}$ comme en $\mathfrak{L}^{\prime}$, il en résulterait que $K$ serait lui même rationnel en $\mathfrak{l}$ et $\mathfrak{l}^{\prime}$, donc 2 -birationnel, ce qui est exclu puisqu'un tel corps est nécessairement totalement imaginaire. En résumé $L$ possède donc deux places 2 -adiques $\mathfrak{L}$ et $\mathfrak{L}^{\prime}$, et $K$ une unique place 2 -adique $\mathfrak{l}$, laquelle se décompose dans l'extension quadratique $L / K$.

Ce point acquis, écrivant la formule des classes ambiges pour les 2-classes (au sens restreint) $\mathcal{C} \ell^{\prime}$ dans l'extension 2-décomposée $L / K$, nous obtenons:

$$
\begin{aligned}
1=\left|\mathcal{C} \ell_{L}^{\prime \operatorname{Gal}(L / K)}\right| & =\left|\mathcal{C} \ell_{K}^{\prime}\right| \frac{2^{n} \prod_{\mathfrak{p} \nmid 2} e_{\mathfrak{p}}}{[L: K]\left(\mathcal{E}_{K}^{\prime}: \mathcal{E}_{K}^{\prime} \cap \mathcal{N}_{L / K}\right)} \\
& =\left|\mathcal{C} \ell_{K}^{\prime}\right| \frac{2^{n+t-1}}{\left(\mathcal{E}_{K}^{\prime}: \mathcal{E}_{K}^{\prime} \cap \mathcal{N}_{L / K}\right)}
\end{aligned}
$$

où $n=[K: \mathbb{Q}]$ est le nombre de places réelles qui se complexifient, $t$ le nombre de places finies qui se ramifient, $\mathcal{E}_{K}^{\prime}$ le groupe des 2-unités (au sens restreint) et $\mathcal{N}_{L / K}$ le groupe des normes locales. Si donc l'extension $L / K$ était 2-ramifiée, nous aurions simultanément $t=0$ (par hypothèse), $\left|\mathcal{C} \ell_{K}^{\prime}\right| \geq 2$ (puisque $K$ posséderait une 2-extension non ramifiée (aux places finies) 2-décomposée non triviale: $L$ ), et $\left(\mathcal{E}_{K}^{\prime}: \mathcal{E}_{K}^{\prime} \cap \mathcal{N}_{L / K}\right)=$ 
$\left(\mathcal{E}_{K}^{\prime}: \mathcal{E}_{K}^{+}\right) \leq 2^{n}$ (puisque les 2-unités normes locales seraient tout simplement celles totalement positives), donc finalement:

$$
\left|\mathcal{C} \ell^{\prime}{ }_{K}\right|=2 \&\left(\mathcal{E}_{K}^{\prime}: \mathcal{E}_{K}^{+}\right)=2^{n}
$$

( $K$ possèderait des 2-unités de toutes signatures).

En particulier le 2-groupe des 2-classes de $K$ au sens ordinaire serait encore d'ordre 2, et l'extension $L / K$ non complexifiée aux places réelles, contrairement au fait que $L$ est totalement imaginaire.

En fin de compte, il vient donc $t \geq 1$; chacun des deux facteurs $\left|\mathcal{C} \ell_{K}^{\prime}\right|$ et $\frac{2^{n+t-1}}{\left(\mathcal{E}_{K}^{\prime}: \mathcal{E}_{K}^{\prime} \cap \mathcal{N}_{L / K}\right)}$ dans la formule des classes ambiges est entier donc vaut 1 ; et il suit $\left|\mathcal{C} \ell_{K}^{\prime}\right|=1$, comme attendu.

Preuve de la proposition: D'après le lemme, nous pouvons supposer $K$ 2-rationnel et $L / K$ 2-décomposée. Ecrivons donc $\mathfrak{L}$ et $\mathfrak{L}^{\prime}$ les deux places 2 -adiques de $L$, notons $2^{a}$ l'ordre du diviseur logarithmique de degré nul $\mathfrak{l}-\mathfrak{l}^{\prime}$ dans le groupe $\tilde{C} \ell_{L}$, et posons $2^{a}\left(\mathfrak{L}-\mathfrak{L}^{\prime}\right)=\operatorname{div}\left(\pi_{L}\right)$, pour un $\pi_{L}$ de $\mathcal{R}_{L}$. Nous pouvons alors écrire le groupe $\mathcal{E}_{L}^{\prime}$ des 2-unités dans $\mathcal{R}_{L}$ comme produit direct

$$
\mathcal{E}_{L}^{\prime}=\tilde{\mathcal{E}}_{L} \pi_{L}^{\mathbb{Z}_{2}}
$$

du sous-groupe $\tilde{\mathcal{E}}_{L}$ des unités logarithmiques de $L$ et du $\mathbb{Z}_{2}$-module monogène engendré par $\pi_{L}$.

Maintenant, puisque $L$ est totalement imaginaire et possède $n=[K$ : $\mathbb{Q}]$ places complexes, $\tilde{\mathcal{E}}_{L}$ est le produit du groupe $\mu_{L}$ des racines de l'unité dans $L$ et d'un $\mathbb{Z}_{2}$-module libre de dimension $n$ (cf. [J1, prop. 3.4]); en particulier il contient $\tilde{\mathcal{E}}_{K}$ avec un indice fini. Mais comme le quotient $\tilde{\mathcal{E}}_{L} / \tilde{\mathcal{E}}_{K}$ est sans torsion (sans quoi nous pourions écrire $L=K[\sqrt{\eta}]$ avec une unité logarithmique $\eta$ de $K$ et l'extension $L / K$ serait 2-ramifiée contrairement aux hypothèses faites), il suit que l'on a l'égalité $\tilde{\mathcal{E}}_{L}=\tilde{\mathcal{E}}_{K}$ (donc en fait $\tilde{\mathcal{E}}_{L}=\mathcal{E}_{K}^{\prime}$ puisque, $K$ n'ayant qu'une seule place 2-adique, les unités logarithmiques de $K$ sont les 2-unités). En résumé nous avons obtenu la décomposition directe:

$$
\mathcal{E}_{L}^{\prime}=\tilde{\mathcal{E}}_{K} \pi_{L}^{\mathbb{Z}_{2}} .
$$

Considérons maintenant le compactifié $\mathcal{R}_{\mathfrak{L}}=\mathcal{R}_{\mathfrak{l}}$ du groupe multiplicatif $L_{\mathfrak{L}}^{\times}=K_{\mathfrak{l}}^{\times}$d'un complété 2-adique de $L$. Le choix d'une uniformisante logarithmique $\pi_{\mathfrak{L}}$ nous permet d'écrire de même $\mathcal{R}_{\mathfrak{L}}=\tilde{\mathcal{U}}_{\mathfrak{L}} \pi_{\mathfrak{l}}^{\mathbb{Z}_{2}}$ à partir cette fois du groupe $\tilde{\mathcal{U}}_{\mathfrak{L}}=\tilde{\mathcal{U}}_{\mathfrak{r}}$ des unités logarithmiques locales. 
Ici encore, l'application de localisation identifie $\tilde{\mathcal{E}}_{K}$ à un module d'indice fini de $\tilde{\mathcal{U}}_{L}$ (du fait de l'égalité des rangs) et finalement à $\tilde{\mathcal{E}}_{K}$ lui même (sans quoi $K$ possèderait une extension 2-décomposée $K[\sqrt{\eta}]$ non triviale engendrée par la racine carré d'une unité logarithmique, i.e. une extension quadratique 2-ramifiée et 2-décomposée, contrairement à la trivialité du groupe $C \ell_{K}^{\prime}$ ).

En fin de compte, on voit que l'application de localisation de $\mathcal{E}_{L}^{\prime}$ dans $\mathcal{R}_{\mathfrak{l}}$ est bijective si et seulement si $\pi_{L}$ est une uniformisante logarithmique; autrement dit que $L$ est 2-birationnel si et seulement si le diviseur logarithmique $\mathfrak{L}-\mathfrak{L}^{\prime}$ est principal:

- si ce n'est pas le cas, le corps $L$ n'est ni 2-birationnel ni logarithmiquement principal;

- si c'est le cas, $L$ est birationnel, le groupe $C \ell_{L}^{\prime}$ est trivial et le groupe $\tilde{C} \ell_{L}$ qui est alors engendré par la classe de $\mathfrak{L}-\mathfrak{L}^{\prime}$ l'est aussi, de sorte que $L$ est logarithmiquement principal.

\section{Démonstration du théorème principal dans le cas modérement ramifié}

Soit $L$ une extension totalement imaginaire d'un corps de nombres totalement réel, ramifiée modérément en au moins une place (finie). D'après la Proposition 5 et le Lemme 6 , nous pouvons supposer $K$ 2-rationnel et $L / K 2$-décomposée, et notre problème est de déterminer sous quelles conditions portant sur la ramification le corps $L$ est logarithmiquement 2-principal.

Notons $G$ le groupe de Galois $\operatorname{Gal}(L / K)$ et écrivons la formule des classes logarithmiques ambiges dans l'extension $L / K$ (cf. $[\mathbf{J} \mathbf{1}$, th. 4.5 et th. 5.3]). Nous obtenons:

$$
\left|\tilde{C} \ell_{L}^{G}\right|=\left|\tilde{C} \ell_{K}\right| \frac{2^{n} \prod \tilde{e}_{\mathfrak{p}}(L / K)}{\left[L^{c}: K^{c}\right]\left(\tilde{\mathcal{E}}_{K}: \tilde{\mathcal{E}}_{K} \cap \mathcal{N}_{L / K}\right)}\left(\mathcal{D}_{L}^{I_{G}} \tilde{\mathcal{P}}_{L}: \tilde{\mathcal{D}}_{L}^{I_{G}} \tilde{\mathcal{P}}_{L}\right),
$$

où $\left|\tilde{C} \ell_{K}\right|$ vaut 1 puisque $K$ est 2-rationnel; $n=[K: \mathbb{Q}]$ est le nombre de places réelles de $K$ complexifiées dans $L$ (elles le sont toutes!); $\tilde{e}_{\mathfrak{p}}(L / K)$ désigne l'indice de ramification logarithmique de la place $\mathfrak{p}$ dans l'extension $L / K$ (lequel coïncide avec l'indice de ramification au sens ordinaire $e_{\mathfrak{p}}(L / K)$, puisque les places 2-adiques, décomposées par hypothèse, sont non ramifiées); le degré $\left[L^{c}: K^{c}\right]=[L: K]$ est égal à 2 ; 
l'indice normique $\left(\tilde{\mathcal{E}}_{K}: \tilde{\mathcal{E}}_{K} \cap \mathcal{N}_{L / K}\right)$ des unités logarithmiques modulo les normes est majoré par $2^{n+1}$ (puisque $\tilde{\mathcal{E}}_{K}$ est le produit de $\{ \pm 1\}$ par un $\mathbb{Z}_{2}$-module libre de rang $\left.n\right)$; et le terme correctif $\left(\mathcal{D}^{I_{G}} \tilde{\mathcal{P}}_{L}: \tilde{\mathcal{D}}^{I_{G}} \tilde{\mathcal{P}}_{L}\right)$ vaut 1 ou 2 (et on sait qu'il vaut 1 lorsque l'extension $L / K$ est primitivement ramifiée).

Il en résulte que si $L$ est 2-logarithmiquement principal, l'extension quadratique $L / K$ n'est ramifiée qu'en une ou deux places.

Reprenons maintenant le même raisonnement à un étage fini $L_{n} / K_{n}$ de la tour cyclotomique: notons $K_{m}$ le $m$-ième étage de la $\mathbb{Z}_{2}$-extension cyclotomique $K^{c}$ de $K$ (c'est encore un corps totalement réel qui est 2-ramifié sur $K$ donc 2-rationnel) et considérons le compositum $L_{m}=$ $K_{m} L$ (qui est le $m$-ième étage de la $\mathbb{Z}_{2}$-extension cyclotomique $L^{c}$ de $L$ ). Observant que $L$ et $L_{m}$ sont simultanément 2-logarithmiquement principaux ou pas (la principalité logarithmique de $L$ comme de $L_{m}$ équivalant à la trivialité du 2-groupe des 2-classes $C \ell_{L^{c}}^{\prime}$ du corps surcirculaire $L^{c}$ (cf. par exemple [JSo])), nous concluons comme précédemment que si $L$ est 2-logarithmiquement principal, l'extension $L_{m} / K_{m}$ n'est ramifiée qu'en une ou deux places.

- Supposons donc L 2-logarithmiquement principal et examinons les deux éventualités:

(i) cas monoramifié: s'il existe une unique place finie ramifiée (modérément) dans $L / K$, elle est forcément imprimitive (sans quoi $L / K$ serait primitivement ramifiée, et $L$ 2-rationnel en vertu du résultat de [GJ], ce qui est exclu $L$ ayant exactement deux places 2-adiques), i.e. décomposée dans $K_{1} / K$; et, quitte à remplacer $K$ par $K_{1}$ et $L$ par $L_{1}$ nous sommes ramenés au:

(ii) cas biramifié: s'il existe exactement deux places finies $\mathfrak{p}$ et $\mathfrak{q}$ ramifiées dans $L / K$, elles sont forcément toutes deux primitives (sans quoi l'une au moins serait décomposée dans $K_{1} / K$ et $L_{1} / K_{1}$ serait ramifiée en trois places au moins).

- Inversement, supposons $L / K$ ramifiée modérément en une unique place $\mathfrak{p}$ semi-primitive, soit en deux places exactement $\mathfrak{p}$ et $\mathfrak{q}$ toutes deux primitives. Alors, quitte à remplacer $L / K$ par $K_{1} / L_{1}$, nous pouvons nous placer dans le second cas. Cela étant, le terme correctif de la formule des classes logarithmique ambiges disparait (l'extension considérée est primitivement ramifiée), et la formule s'écrit:

$$
\left|\tilde{C} \ell_{L}^{G}\right|=\frac{2^{n+1}}{\left(\tilde{\mathcal{E}}_{K}: \tilde{\mathcal{E}}_{K} \cap N_{L / K}\right)}=\frac{2^{n+1}}{\left(\mathcal{E}_{K}^{\prime}: \mathcal{E}_{K}^{\prime} \cap N_{L / K}\right)} .
$$


D'autre part nous savons déjà, puisque $K$ est 2-rationnel, qu'il contient des 2-unités de toutes signatures. En d'autres termes, les 2-unités totalement positives (i.e. celles qui sont normes locales aux places infinies dans l'extension $L / K)$ forment un sous-groupe d'indice $2^{n}$ dans $\mathcal{E}_{K}^{\prime}$. Montrer que $\tilde{C} \ell_{L}$ est trivial revient donc à trouver une 2-unité totalement positive qui ne soit pas norme locale en $\mathfrak{p}$ (ou en $\mathfrak{q}$, ce qui est équivalent d'après la formule du produit). Or nous connaissons une telle 2-unité $\varepsilon$ : le premier étage $K_{1}$ de la $\mathbb{Z}_{2}$-extension cyclotomique $K^{c}$ de $K$ est précisément de la forme $K[\sqrt{\varepsilon}]$ pour une 2-unité $\varepsilon \gg 0$ qui n'est pas un carré dans $K_{\mathfrak{p}}^{\times}$ (puisque $\mathfrak{p}$ ne se décompose pas dans $K_{1} / K$ ) donc qui n'est pas norme locale en $\mathfrak{p}$ dans l'extension quadratique $L / K$ ramifiée en $\mathfrak{p}$; ce qui achève la démonstration.

Remarque. Pour $K=\mathbb{Q}$, le résultat obtenu redonne naturellement la classification des corps quadratiques imaginaires 2-logarithmiquement principaux $\mathbb{Q}[\sqrt{-d}]$ (avec ici $d \equiv-1[\bmod 8]$ compte tenu de la contrainte de 2-décomposition) établie dans [So].

\section{Bibliographie}

[GJ] G. Gras et J.-F. Jaulent, Sur les corps de nombres réguliers, Math. Z. 202(3) (1989), 343-365.

[J1] J.-F. JaUlent, Classes logarithmiques des corps de nombres, $J$. Théor. Nombres Bordeaux 6(2) (1994), 301-325.

[J2] J.-F. Jaulent, Théorie $\ell$-adique globale du corps de classes, $J$. Théor. Nombres Bordeaux 10 (1999), 355-397.

[JN] J.-F. Jaulent et T. NGuyen Quang Do, Corps p-rationnels, corps p-réguliers, et ramification restreinte, J. Théor. Nombres Bordeaux 5(2) (1993), 343-363.

[JSa] J.-F. Jaulent et O. Sauzet, Pro- $\ell$-extensions de corps l-rationnels, J. Number Theory 65(2) (1997), 240-267.

[JSo] J.-F. Jaulent et F. Soriano, Sur les tours localement cyclotomiques, Arch. Math. (Basel) 73(2) (1999), 132-140.

[So] F. Soriano, Classes logarithmiques ambiges des corps quadratiques, Acta Arith. 78(3) (1997), 201-219.

[MN] A. Movahhedi et T. Nguyen Quang Do, Sur l'arithmétique des corps de nombres p-rationnels, in: "Sém. Th. Nombres Paris 1987-88", Prog. Math. 81, Birkhäuser Boston, Boston, MA, 1990, pp. $155-200$.

[W1] K. WingBerG, On Galois groups of $p$-closed algebraic number fields with restricted ramification, J. reine angew. Math. 400 (1989), 185-202. 
[W2] K. WingBerg, On Galois groups of $p$-closed algebraic number fields with restricted ramification II, J. reine angew. Math. 416 (1991), 187-194.

Institut de Mathématiques

Université Bordeaux I

351 , cours de la libération

F-33405 Talence Cedex

France

E-mail address: jaulent@math.u-bordeaux.fr

E-mail address: sauzet@math.u-bordeaux.fr

Primera versió rebuda el 20 de juliol de 1999, darrera versió rebuda el 8 d'octubre de 1999. 